\title{
The Definition of Electrochromism
}

\author{
Stephen Fletcher \\ Prof S. Fletcher, Department of Chemistry, Loughborough University, \\ Ashby Road, Loughborough, Leicestershire LE11 3TU, UK. \\ E-mail: stephen.fletcher@Lboro.ac.uk.
}

Submitted: 27 Aug 2015. Revised: 17 Sept 2015.

Manuscript number: JSEL-D-15-00622 • DOI: 10.1007/s10008-015-3039-9

\section{Dedication}

This paper is dedicated to the memory of Prof Roger J. Mortimer (17 January 1956 - 02 May 2015), electrochemist and friend.

\section{Keywords}

Electrochromism; Photochromism; Feynman Diagrams; Liquid Crystals; Conducting Polymers;

\begin{abstract}
Many experimental studies of electrochromism have been reported in the scientific literature, but the terminology is still not settled, particularly for the case in which an electrochromic material forms the load in an electrical circuit. In the present work, two new terms are introduced to help clarify this situation. These terms are "dielectric electrochromism" and "faradaic electrochromism". The first term applies to the case where an applied electric current causes a change in the energy of the electronic states in the material, while the second term applies to the case where an applied electric current causes a change in the occupation number of the electronic states in the material. Precise definitions are given of both terms.
\end{abstract}

\section{Introduction}

The history of electrochromism spans more than 100 years and its origins are far from clear. However, a functioning electrochromic device was clearly described in the US patent "Electrographic Display Apparatus and Method" granted to Thomas Edison's chief engineer, Miller Reese Hutchison, in 1913 [1]. This invention was based on the electrolysis of a liquid film of electrolyte solution. More than four decades later, in 1957, a solid-state device capable of 
modulating the intensity of a light beam was patented by Kurt Lehovec, and this may be regarded as the beginning of the modern era [2]. Lehovec achieved his historic breakthrough by injecting charge carriers into a thin slice of semiconductor. Shortly afterwards, in 1961, the word "electrochromism" appeared for the first time, in an article by John R. Platt [3]. Subsequently, more than a thousand patents have been granted in the US on the topic of electrochromic devices.

In the present work, we define Electrochromism in the following way:

Electrochromism is an electro-optical property of condensed phase matter, in which an electric current induces a change in either the energy or the occupation number of the internal electronic states of the matter, such that a change is also induced in the absorption, scattering, or polarization of incident radiation.

In the most well-known manifestation of electrochromism, transmitted or reflected radiation is observed to have a different power spectral density than it had before the electrical perturbation was applied to the system. In less wellknown manifestations of electrochromism, transmitted or reflected radiation may also have a different power angular density, and/or a different state of polarization, than they had before the electrical perturbation was applied to the system.

It is instructive to consider the simplest possible Feynman diagram of the electrochromic effect, which is shown in Fig. 1. In this diagram, the input electron is denoted by $e_{\text {in }}$ and the output electron is denoted by $e_{\text {out }}$. Inside the electrochromic material, the input electron perturbs one of the bound state electrons $e_{\chi}$ causing it to scatter (change energy or enter a new electronic state). This is labelled $e_{\chi}^{\prime}$. The latter is then scattered a second time by interaction with an incident photon $\gamma_{\text {in }}$. Finally, the twice-scattered electron $\left(e_{\chi}^{\prime \prime}\right)$ emits a photon $\gamma_{\text {out }}$ and is scattered yet a third time as $e_{\chi}^{\prime \prime \prime}$. Since the output photon is characterized by three parameters (energy, direction of travel, and polarization state) it follows that all three of these parameters may change during the electrochromic process. 


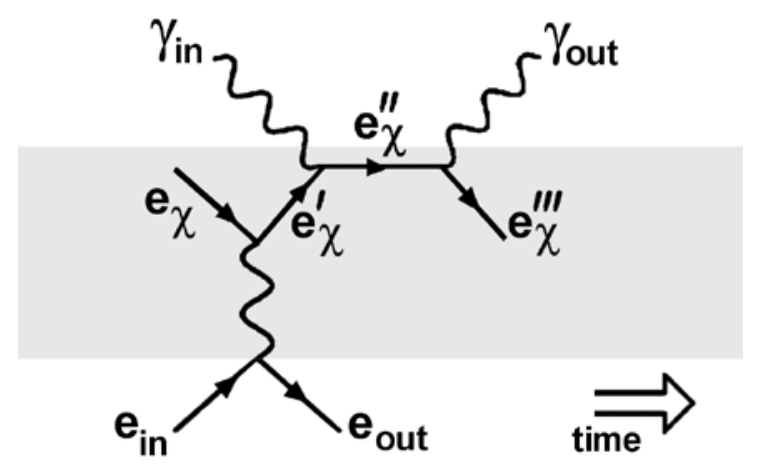

Fig. 1 The simplest possible Feynman diagram of the electrochromic effect. Many more complex diagrams, involving different charge carriers, arise in nature. (For notation see text.)

\section{The Definitions}

Although many experimental studies of electrochromism have been carried out by researchers over the years, the terminology is still not settled, particularly in the case where the electrochromic material forms the load in an electrical circuit [4]. For example, terms such as "redox electrochromism", and "visible electrochromism", are still in widespread use, while older terms such as "persistent electrochromism" and "electrochemichromism" may be found in the patent literature. An unfortunate result of this multiplicity of terms is a lack of clarity regarding the essential and non-essential features of the phenomenon. For example, when viewed from the perspective of quantum mechanics, there is clearly no requirement that the changes in electronic states should be reversible, even though this is highly desirable in a functioning device. Similarly, there is no requirement that the changes in electronic states should be persistent, even though this is also a desirable feature of a functioning device. Finally, there is no need to restrict the definition of electrochromism to visible light, since the corresponding ranges of frequencies and wavelengths (approximately $v=750-375 \mathrm{THz}, \lambda=400-$ $800 \mathrm{~nm}$ in air) are arbitrarily determined by the spectral resolution of the human eye.

As a contribution to the development of an improved notation in this field, we here suggest two new terms (dielectric electrochromism and faradaic electrochromism) which avoid previous ambiguities, and which accord with the quantized nature of the internal electronic states in the condensed phase (liquid or solid). Crucially, these new definitions take into account the fact that an observed change in power spectral density ("absorption") or power angular density ("scattering") or spin ("polarization state") may be due to two distinct causes: (i) a change in the energy of the electronic states in a system, or (ii) a 
change in the occupation number of the electronic states in a system. In the mathematical formulation of quantum mechanics the energy and the occupation number are separate variables, and hence it is rational to deal with them separately.

\section{Special Case I. A Change in the Energy of the Electronic States of an Electrochromic Material.}

A change in the energy of an electronic state of a material can readily be brought about by a change in the internal electric field. Here, we are concerned with the case where a change in the internal electric field is caused by a flow of electric current. Since the study of electric fields in matter is the province of dielectric science, this immediately suggests the following definition:

A material acting as a load in an electrical circuit is said to exhibit dielectric electrochromism if the induced change in energy of its internal electronic states causes a change in the absorption, scattering, or polarization of incident radiation.

The Stark effect (which describes the splitting of the spectral lines of atoms and molecules by an applied electric field) provides a good example of dielectric electrochromism. On the above definition, Liquid Crystals also exhibit dielectric electrochromism, even though their optical output commonly does not involve a colour change. For example, in many commercial liquid crystal devices, the positions, orientations, and shapes of constituent molecules are distorted by the applied electric field, causing changes to the intensity and polarization of the incident radiation, but not the colour. Richard Williams of the RCA Corporation patented the first display based on liquid crystals in 1967 [5].

\section{Special Case II. A Change in the Occupation Number of the Electronic States of an Electrochromic Material.}

A change in the occupation number of an electronic state of a material is known as electron transfer. Electron transfer is a special type of quantum transition in which an electron delocalizes from one bound state, and localizes in another bound state, thereby inducing a change in the occupation number of both states [6]. Since the study of electron transfer is the province of faradaic electrochemistry, this immediately suggests the following definition: 
$\square$ A material acting as a load in an electrical circuit is said to exhibit faradaic electrochromism if the induced change in occupation number of its internal electronic states causes a change in the absorption, scattering, or polarization of incident radiation.

Prussian blue is a good example of a faradaic electrochromic material. Other well-known examples include transition-metal phthalocyanines, the viologens, conducting polymers, and innumerable redox indicators.

\section{Afterword: Some Disambiguations}

To clarify the above definitions, we add the following remarks.

(1) Etymology. "Electrochromism" is a compound word derived from two Latinized Greek roots, elektro- and khroma. The first root refers to electricity while the second root refers to colour. As in the case of the word "chromatography", the word "electrochromism" was originally applied only to systems demonstrating visible changes of colour, but its area of application has subsequently broadened to include colourless systems.

(2) Scattering, in its broadest sense, refers to electromagnetic interactions by which matter is excited to radiate by an external source of photons. On this view, reflection, refraction, and even transmission of electromagnetic waves are all types of scattering. In a narrower sense, which we adopt in the present definitions, the word "scattering" is restricted to processes not involved in specular reflection, refraction, and transmission.

(3) Displacement Current. The electric current that induces a change in the internal electronic states of the electrochromic material may be a regular current or a displacement current. In the latter case, dielectric electrochromism may be observed inside insulators.

(4) Electroluminescence occurs when a material actually generates photons in response to the passage of an electric current. By contrast, electrochromism involves the absorption, scattering or polarization of incident photons generated elsewhere. Other forms of photon emission may result from the action of heat (incandescence), or from chemical reactions (chemiluminescence).

(5) Colour Space. The power spectral density can be transformed mathematically into measures of hue, saturation, and brightness, which in turn can be correlated with the subjective experience of colour detected by the human eye [7]. 
(6) Reversibility and persistence and visible colours are all desirable attributes of electrochromic devices, but they are not necessary features of electrochromism.

(7) A Load is any component of an electrical circuit that stores or dissipates electric power.

(8) Redox electrochromism is a term that is sometimes used to describe faradaic electrochromism. However, the word "redox" carries the inference of "reversibility", which is unnecessarily restrictive. The use of this term is therefore discouraged.

(9) Photochromism. It has long been recognized that the definitions of electrochromism and photochromism are in some sense "dual" to each other. This being the case, it is particularly unfortunate that the IUPAC "gold book" [8] defines photochromism in the following way:

"Photochromism is the reversible transformation of a molecular entity between two forms, $A$ and $B$, having different absorption spectra, induced in one or both directions by absorption of electromagnetic radiation."

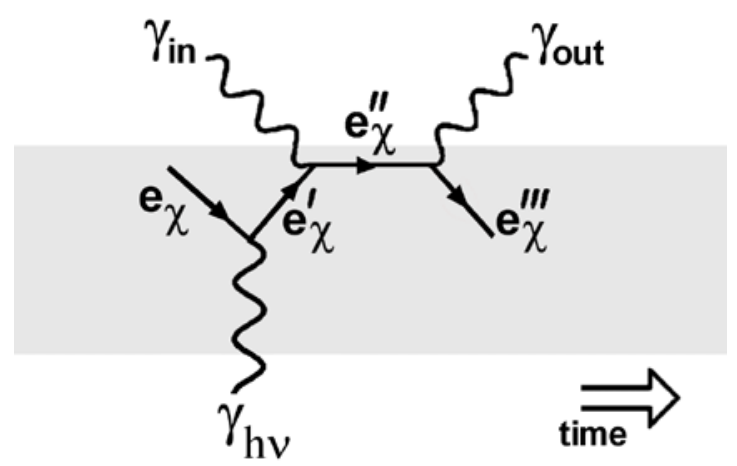

Fig. 2 The simplest possible Feynman diagram of the photochromic effect.

(For notation see text.)

In this definition we immediately note the arbitrary restrictions to reversible systems, to absorption spectra, and to molecular entities, all of which are unnecessary. Referring to the Feynman diagram shown in Fig. 2 we can immediately suggest a better definition:

Photochromism is an optical property of condensed phase matter, in which a flux of photons $\left(\gamma_{\mathrm{hv}}\right)$ induces a change in the internal electronic states of the 
matter, such that a change is also induced in the absorption, scattering, or polarization of incident radiation.

(10) Further reading. Commercial applications of electrochromic materials, and their performance parameters, are briefly discussed in Roger Mortimer's entry "Electrochromism" in the Electrochemical Dictionary [9].

\section{References}

[1] Hutchison MR, Electrographic Display Apparatus and Method, US Patent 1,068,774 (29 July 1913)

[2] Lehovec K, Photon Modulation in Semiconductors, US Patent 2,776,367 (01 Jan 1957)

[3] Platt JR (1961) Electrochromism, a Possible Change of Color Producible in Dyes by an Electric Field. J Chem Phys 34:862-863

[4] Electrochromism and Electrochromic Devices, by Monk PMS, Mortimer RJ, Rosseinsky DR, Cambridge University Press, Cambridge (2007). [ISBN 9780-521-82269-5]

[5] Williams R, Electro-optical Elements Utilizing an Organic Nematic Compound, US Patent 3,322,485 (30 May 1967)

[6] Fletcher S (2010) The Theory of Electron Transfer. Journal of Solid State Electrochemistry, 14:705-739

[7] Proceedings of the Commission Internationale de l'Éclairage, Session de 1931. Edited by Walter Stanley Stiles, Cambridge University Press, Cambridge (1932).

[8] IUPAC Compendium of Chemical Terminology, 2nd ed. Compiled by McNaught $A D$ and Wilkinson A, Blackwell Scientific Publications, Oxford (1997). [ISBN 0-86542-6848]

[9] Mortimer RJ, "Electrochromism" in Electrochemical Dictionary. 2nd ed. Edited by Bard AJ, Inzelt G, and Scholz F, Springer Science \& Business Media, Berlin Heidelberg, pp. 271-272, (2012) 


\section{Figure Captions}

Fig. 1 The simplest possible Feynman diagram of the electrochromic effect. Many more complex diagrams, involving different charge carriers, arise in nature. (For notation see text.)

Fig. 2 The simplest possible Feynman diagram of the photochromic effect. (For notation see text.) 